\title{
Über die Einwirkung metallischen Molybdäns und Wolframs auf Lösungen von Silber, Gold und anderen Metallen.
}

\author{
Von \\ Edgar F. SMith.
}

Wenn metallisches Molybdän, welches durch Reduktion von Molybdäntrioxyd in einem Wasserstoffstrom unter starker Erhitzung dargestellt war, zu einer Lösung von salpetersaurem Silber gegeben wird, tritt sofort eine Abscheidung von glänzenden metallischen Silberblättchen ein, und die Lösung trübt sich infolge der Ausscheidung von Molybdänsäure. Um dies zu verhindern, wurde zu der Lösung des salpetersauren Silbers in Wasser Ammoniak gefügt und das erhaltene Silberoxyd in geringem Überschufs von Ammoniak aufgelöst. Bei Eintragen des Molybdäns in die auf diese Weise hergestellte Lösung löste es sich fast augenblicklich auf, und ein reichlicher Niederschlag von Silber schied sich aus, welcher beim Reiben an den Seiten des Becherglases vermittelst eines Glasstabes hohen Glanz zeigte, sofort niederfiel und beim Stehenlassen eine krystallinische Struktur annahm. Folgende Bestimmung beweist, dafs der auf diese Weise erhaltene Silberniederschlag quantitativ ist:

1. $0.1044 \mathrm{~g}$ metallischen Molybdäns wurde zu einer Lösung salpetersauren Silbers in Wasser gegeben, welche etwa ein Gramm Silber und genügend Ammoniak enthielt, um das Silberoxyd niederzuschlagen und zugleich aufzulösen. $\mathrm{Zu}$ dieser Lösung wurden $40 \mathrm{ccm}$ destillierten Wassers gegeben und die Mischung vier Stunden lang stehen gelassen. Der entstandene Silberniederschlag wurde abfiltriert, gewaschen, geglüht und gewogen. Derselbe wog $0.7005 \mathrm{~g}$.

$$
\begin{aligned}
& 0.1044 \div 96=.00108=1 \\
& 0.7005 \div 108=.00648=6 .
\end{aligned}
$$

Diesem Verhältnis gemäls sollten $0.1044 \mathrm{~g}$ Molybdän $0.7047 \mathrm{~g}$ Silber niederschlagen. Der Unterschied zwischen dem in diesem Falle erhaltenen Resultat und der theoretischen Zabl beträgt also $0.5 \%$.

Bei zwei weiteren angestellten Bestimmungen wurde das Silber zunächst als Chlorsilber niedergeschlagen, das Präcipitat gewaschen und dann in Ammoniak aufgelöst. In folgendem sind die Einzelheiten des Verfahrens angegeben: 
2. $0.1035 \mathrm{~g}$ metallischen Molybdäns zu einer Lösung von Chlorsilber in Ammoniak gegeben, welche mit $3 \mathrm{ccm}$ Ammoniak im Überschufs und $60 \mathrm{ccm}$ Wasser versetzt war, ergaben $0.6926 \mathrm{~g}$ metallischen Silbers:

$$
\begin{gathered}
0.1035 \div 96=.00107=1 \\
0.6926 \div 108=.00641=6 \\
\text { Mo }: 6 \mathrm{Ag}=0.1035: \times(=0.6986 \mathrm{~g}) .
\end{gathered}
$$

3. $\quad 0.1032 \mathrm{~g}$ Molybdän wurden zu einer Lösung von Chlorsilber in Ammoniak gegeben - letzteres in gerade genügender Menge, um das Chlorsilber in Lösung zu halten - und dann mit $50 \mathrm{ccm}$ Wasser verdünnt. Der erhaltene Silberniederschlag wog $0.6937 \mathrm{~g}$. Dieses Resultat ergiebt ébenfalls das Verhältnis - $-1 \mathrm{M}_{0}: 6 \mathrm{Ag}$, und die dem zugefügten Molybdänquantum entsprechende Silbermenge würde $0.6966 \mathrm{~g}$ betragen.

In der ersten Bestimmung (1) wirkte das Molybdän mit einer Wertigkeit von sechs, ebenso in 2 und 3 , und da diese letzteren das Silber als Chlorsilber enthielten, ging das Molybdän wahrscheinlich als Hexachlorid $\left(\mathrm{MoCl}_{6}\right)$ in Lösung. Diese Annahme scheint um so mehr gerechtfertigt, als das Molybdän aus Lösungen von Gold. chlorid das Gold sofort niederschlägt, und die Bestimmungen, in welchen das niedergeschlagene Gold gewogen wurde, das Verhältnis 1Mo : $2 \mathrm{Au}$ ergaben. Die über dem niedergeschlagenen Silber stehende Flüssigkeit, in welcher letzteres als Chlorid gegenwärtig ist, nahm eine bräunliche Färbung an, und die Farbe der Lösung, aus welcher das Gold niedergeschlagen wurde, ging von einer gelben in eine tiefbraune Färbung über.

Fein verteiltes Wolfram, welches ebenfalls durch die Reduktion des Oxyds in Wasserstoffstrom dargestellt worden war, schlug das Silber aus einer Lösung des Nitrats, welche einen geringen Überschuls von Ammoniak enthielt, sofort, und zwar vollständig nieder. Bei den zwei ersten Bestimmungen wurden die Lösungen sieben Stunden lang stehen gelassen, ehe das gefällte Silber abfiltriert wurde. Das erhaltene Metall war von völlig weilser Farbe.

1. $0.1037 \mathrm{~g}$ metallischen Wolframs schlugen nieder $0.365 \tilde{7} \mathrm{~g}$ Silber:

$$
\begin{gathered}
0.1037 \div 184=.00056=1 \\
0.3657 \div 108=.00337=6 \\
\mathrm{~W}: 6 \mathrm{Ag}=0.1037: \times(0.3652 \mathrm{~g}) . \quad \text { Gefunden }: 0.3657 .
\end{gathered}
$$

2. $0.1030 \mathrm{~g}$ metallischen Wolframs gaben $0.3614 \mathrm{~g}$ Silber. Dem Verhältnis 1:6 gemäfs sollte das erhaltene Silber ein Gewicht von $0.3627 \mathrm{~g}$ gegeben haben. 
3. $0.1037 \mathrm{~g}$ Wolfram wurden zu einer Lösung von salpetersaurem Silber gegeben, welche aufser dem zur Fällung und Lösung des Silberoxyds nötigen Ammoniak $10 \mathrm{ccm}$ des letzteren und $20 \mathrm{ccm}$ destillierten Wassers enthielt. Der erhaltene Silberniederschlag wog 0.3653 g. Berechnet $\mathrm{Ag}=0.3652 \mathrm{~g}$.

4. $0.1037 \mathrm{~g}$ Wolfram, $10 \mathrm{ccm}$ salpetersauren Silbers (Silbergehalt $0.6000 \mathrm{~g}), 10 \mathrm{ccm}$ Ammoniak im Überschuls and $20 \mathrm{ccm}$ Wasser ergaben $0.3617 \mathrm{~g}$ Silber.

5. $0.2074 \mathrm{~g}$ Wolfram, $20 \mathrm{ccm}$ salpetersauren Silbers, $20 \mathrm{ccm}$ Ammoniak im Überschufs und $40 \mathrm{ccm}$ Wasser ergaben $0.7314 \mathrm{~g}$ Silber. Berechnet $0.7304 \mathrm{~g}$ Silber.

6. $\quad 0.2073 \mathrm{~g}$ Wolfram ergaben, unter sonst gleichen Bedingungen wie in $5,0.7267 \mathrm{~g}$ Silber statt $0.7300 \mathrm{~g}$ berechnet.

7. $0.1037 \mathrm{~g}$ Wolfram, $10 \mathrm{ccm}$ salpetersauren Silbers, $10 \mathrm{ccm}$ Ammoniak im Überschufs und $25 \mathrm{ccm}$ Wasser gaben $0.3661 \mathrm{~g}$ Silber statt $0.3652 \mathrm{~g}$.

8. $0.1037 \mathrm{~g}$ Wolfram, $10 \mathrm{ccm}$ salpetersauren Silbers, $3 \mathrm{ccm}$ Ammoniak im Überschufs und $20 \mathrm{ccm}$ Wasser gaben $0.3645 \mathrm{~g}$ Silber statt $0.3652 \mathrm{~g}$.

Es wurden zwei weitere Bestimmungen mit diesem Metall und Silber gemacht, das letztere zunächst als Chlorsilber gefällt, gründlich mit Wasser gewaschen und dann in gerade genügender Menge Ammoniak gelöst, worauf die Lösung mit Wasser verdünnt wurde:

9. $0.1012 \mathrm{~g}$ Wolfram schlugen $0.3576 \mathrm{~g}$ Silber nieder. Berechnet $0.3564 \mathrm{~g}$.

10. $\quad 0.1024 \mathrm{~g}$ Wolfram schlugen $0.3592 \mathrm{~g}$ Silber anstatt $0.3606 \mathrm{~g}$ desselben nieder.

Metallisches Wolfram schlug Gold nur sehr langsam aus seinen Chloridlösungen nieder; in einer alkalischen Lösung bildete sich der Niederschlag jedoch viel schneller.

Da eine Verunreinigung sowohl von Wolfram als Molybdän durch Stickstoff nicht ausgeschlossen war, insofern beide Metalle aus Ammonsalz dargestellt waren, beabsichtigen wir eine Wiederholung der Versuche mittelst auf anderem Wege erhaltener Metalle, um so das Verhältnis von Silber und Gold zu den beiden Metallen endgültig festzustellen.

Wolfram und Molybdän wirken nicht ein auf neutrale, alkalische oder saure Lösungen von salpetersaurem Blei.

Wenn Molybdän zu einer neutralen Lösung von Quecksilberchlorid gegeben wird, entsteht sofort ein Niederschlag von weifsem 
Quecksilberchlorür, welcher beim Stehenlassen, mit dem Metall im Überschufs, langsam in metallisches Quecksilber übergeht.

Metallisches Wolfram reduziert Quecksilberchlorid nur sehr langsam zu Quecksilberchlorür.

Molybdän schlug aus einer Lösung von Jodkaliumquecksilber in Gegenwart von Natriumhydroxyd allmählich graues Quecksilber nieder. Wolfram verursachte unter gleichen Bedingungen eine ähnliche, aber unvollständige Reduktion des Quecksilberchlorids zu Quecksilberchlorür.

Wenn Molybdän zu einer Lösung von schwefelsaurem Quecksilberoxydul gegeben wird, so schlägt sich metallisches Quecksilber nieder, und die darüber stehende Flüssigkeit nimmt eine tiefblaue Färbung an. Bei Anwendung von Wolfram war diese Reduktion äufserst unvollständig oder trat gar nicht ein.

Molybdän, sowie Wolfram schlugen beide metallisches Kupfer aus Kupfersalzlösungen nieder, wiewohl die Ausscheidung keine vollkommene war. Lösungen von Wismuth oder Cadmium blieben in Gegenwart beider Metalle unverändert, während Platin-, Palladiumund Rhodiumchlorid nur teilweise von denselben reduziert wurden.

Universität von Pennsylvanien, Philadelphia. 